\title{
Status of PLS-II Synchrotron Light Source
}

\author{
H.S. Lee ${ }^{1}$
}

Pohang Accelerator Laboratory

Pohang, Kyungbuk 790-784, KOREA

E-mail: lhs@postech.ac.kr

\section{S.H. Shin, S.J. Kwon, D.T. Kim, D.E. Kim, M.G. Kim, S.H. Kim, S.C. Kim, J.M. Kim, C.B. Kim, B.Y. Park, S.S. Park, S.J. Park, Y.S. Park, Y.G. Son, J.C. Yoon, B.J. Lee, E.H. Lee, G.H. Kim, S.D. Jang, Y.D. Joo, J.Y. Choi, T.G. Ha, W.H. Hwang, I.M. Hwang, J.W. Lee, J. Y. Hwang}

Pohang Accelerator Laboratory

Pohang, Kyungbuk 790-784, KOREA

\begin{abstract}
Pohang light source (PLS) had been operated for 14 years successfully. The number of beamlines of PLS was two at the beginning stage. The number of beam-lines has been increased two or three every year. And the total beam-lines in operation became 28 in 2010 and 3199 users had visited PAL for their experiments [1]. The user community gradually extended and they became to require brighter beam to see small signal from a sample. To meet this requirement of the user community, the PLS machine was upgraded as PLS-II. The main goals of the PLS-II machine are to increase the beam energy from 2.5 to $3 \mathrm{GeV}$, and to increase the number of insertion device (ID) by the factor of two (20 IDs). This PLS-II upgrade project had been completed in 2011 and user service has been started in 2012. We will report the current status of PLS-II machine in this paper.
\end{abstract}

1

Heung-Soo Lee, Email:Ihs@ postech.ac.kr 
The 21 st International Workshop on Vertex Detectors 16-21 September 2012

Jeju, Korea

Jeju, Korea 


\section{Introduction}

PLS consists of a storage ring and a linear accelerator (Linac) as a full energy injector without any booster ring. PLS project was launched on April 1, 1988 and was completed in 1994. After commissioning for about a year, we started user service on September 1, 1995. During user service for five years, many x-ray users wanted much higher flux of x-ray beam to do experiments with samples which have weak signals. So we had ramped up the energy of the storage ring from 2.0 to $2.5 \mathrm{GeV}$. After a couple of years late, we had begun to inject $2.5 \mathrm{GeV}$ electron beam in the storage ring directly by installing a modulator and a klystron for feeding $\mathrm{RF}(2856 \mathrm{MHz})$ in two accelerating structures.

Table 1. PLS chronology

\begin{tabular}{|l|l|}
\hline Event & Date \\
\hline Project start & Apr. 1 1988 \\
\hline Ground-breaking & Apr. 1 1991 \\
\hline 2 GeV linac commissiong & June 30, 1994 \\
\hline Storage ring commissioning & Dec. 24, 1994 \\
\hline User service start & Sept. 1, 1995 \\
\hline Energy ramping to 2.5 GeV & Sept. 1, 2000 \\
\hline 2.5 GeV injection & Nov. 1, 2002 \\
\hline 3GeV linac rf conditioning & May 1, 2011 \\
\hline 3GeV linac commissioning & June 1, 2011 \\
\hline 3GeV storage ring commissioning & July 1, 2011 \\
\hline User service start again & March 21, 2012 \\
\hline
\end{tabular}

After seven years operation for users, we decided to upgrade PLS for three years. So, this project was mapped out detailed action plans such as two year preparation and a year machine shutdown. We changed the lattice of the storage ring and the number of the insertion devices from 10 to 20. Also the beam energy of the new storage ring was increased from 2.5 to $3.0 \mathrm{GeV}$ [2]. And user service started on March 21, 2012 as described in table 1.

\section{Linac Upgrade}

The Linac building has a limited length because original Linac energy was a $2.0 \mathrm{GeV}$. The Linac has 11 klystrons for feeding RF to 42 accelerating structures. Each klystron feed RF to four accelerating structures like as figure 1. Averaged accelerating gradient is about $16 \mathrm{MV} / \mathrm{m}$ in the accelerating structure at that time. After we increased the energy of Linac from 2.0 to 2.5 $\mathrm{GeV}$ by adding two accelerating structures and by elevating the operating voltages of the klystrons, averaged accelerating gradient became to $19 \mathrm{MV} / \mathrm{m}$. 


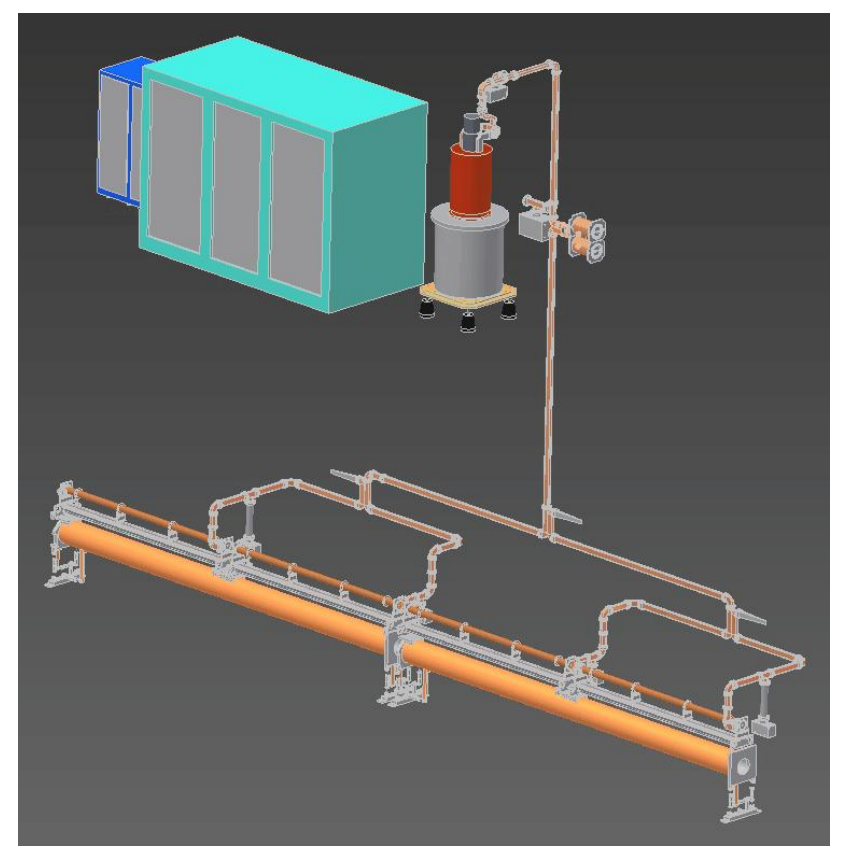

Figure 1. Layout of the RF system of the PLS Linac.

We installed two more accelerating structures for PLSII Linac. There is no more remained space length in Linac after installing these structures. Finally, 46 accelerating structures have been used for a $3.0 \mathrm{GeV}$ Linac. In this case, the required averaged accelerating gradient is about $22 \mathrm{MV} / \mathrm{m}$. If the maximum output power of an $80 \mathrm{MW}$ klystron is fed into four accelerating structures through a SLED which has the beam energy gain of 1.5, the available maximum accelerating gradient is about $22 \mathrm{MV} / \mathrm{m}$. In such case, it is impossible to operate all klystrons at the maximum out-put power. Hence, we added four klystrons by separating the accelerating structures as shown in figure 2 to get the electron beam of $3.0 \mathrm{GeV}$ safely.

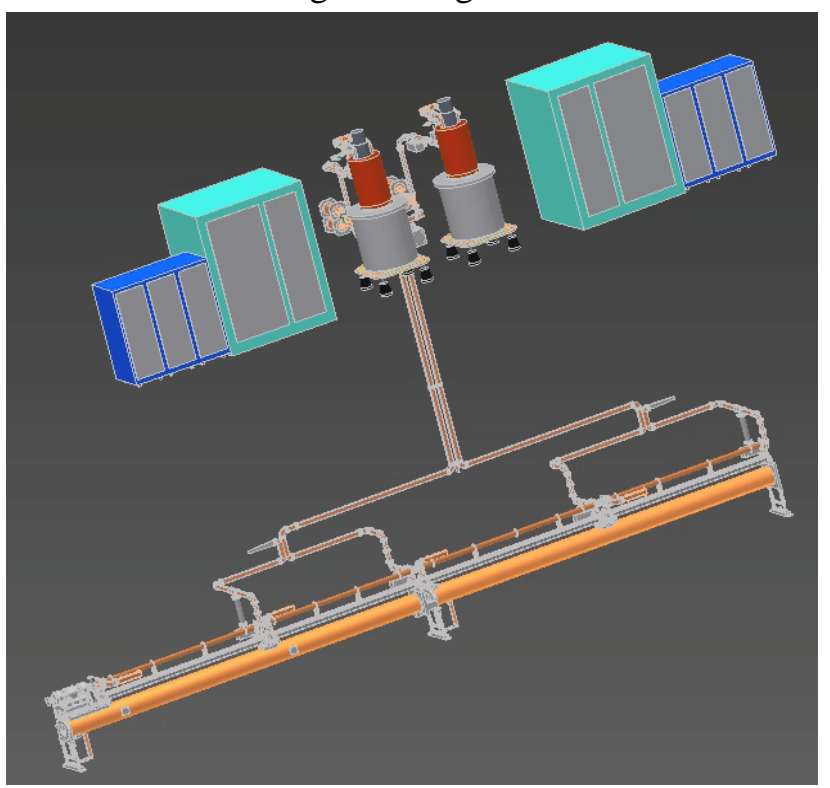

Figure 2. Layout of a modified RF system for the PLSII Linac. 
As a result, PLSII Linac is composed of 16 klystrons, 15 SLEDs and 46 accelerating structures. Eight klystrons feed RF to two accelerating structures through SLEDs respectively and the others except the injector klystron supply RF to four accelerating structures through SLEDs respectively. And seven existing modulators so called "Line Type Modulator" [3] supply electric power to the klystrons which supply RF to four accelerating structures. The other eight modulators called "Invert Type Modulator" were installed which is more compact in size than the line-type due to the limited space in the Linac gallery.

\section{Storage Ring Upgrade}

The main goals for upgrading the storage ring are energy and current increasing, emittance reducing, number of insertion device increasing and top-up operation as shown in table 2 . The storage ring building of PLS had a limitation in making $5.8 \mathrm{~m}$ long straight sections for 20 insertion devices. So, half of the straight sections are $5.8 \mathrm{~m}$ long and the rest about half in length. And we installed 24 gradient dipole magnets so that each magnet realize 15 degree bend in the electron beam trajectory.

Table 2. Main goals of PLSII

\begin{tabular}{|l|l|l|}
\hline Parameter & PLS & PLSII \\
\hline Beam energy $(\mathrm{GeV})$ & 2.5 & 3.0 \\
\hline Current $(\mathrm{mA})$ & 200 & 400 \\
\hline Emittance (nm-rad) & 18.9 & 5.8 \\
\hline Top-up operation mode & No & Yes \\
\hline No. of insertion device & 10 & 20 \\
\hline
\end{tabular}

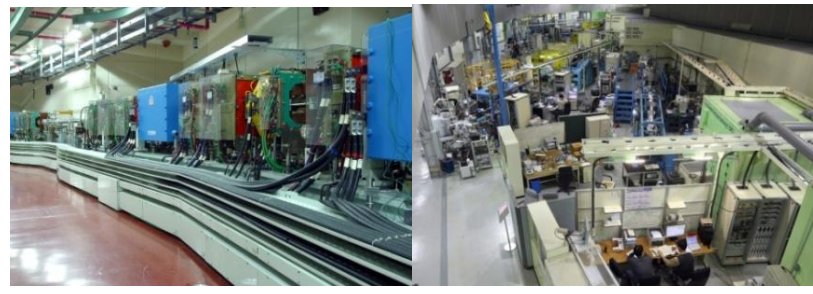

Figure 3. View of the PLS storage ring before upgrading (left) and the experimental hall (right) on December in 2010.

Figure 3 shows the view of the PLS storage ring and the experimental hall. We finished user service of PLS on December 10, 2010 and started to dismantle it. And we finished these works within a month and a half completely as shown in figure 4 . The left picture is the view of the storage ring and the right is the sight of the experimental hall for beam-lines. 


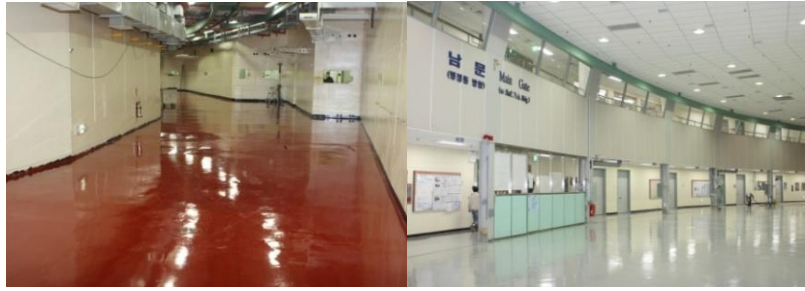

Figure 4. View of the storage ring (left) and the experimental hall (right) after dismantling.

After dismantling the machine, we started to do reinstallation on January 25, 2011 and finished it on June 25,2011 . The left picture in figure 5 is the sight of the storage ring and the right picture is the view of the experimental hall.

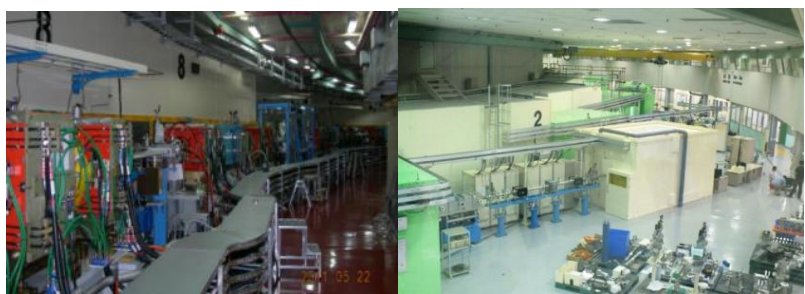

Figure 5. View of the storage ring (right) and the experimental hall (left) after reinstallation.

\section{Commissioning}

We began the commissioning of PLSII Linac on May 23, 2011 to keep the commissioning schedule of the storage ring. We achieved $3.0 \mathrm{GeV}$ on June 24, 2011. So beam transport line commissioning was begun on June 25, 2011. And the first turn of the storage ring was achieved on July 1, 2011. We had a big accident during the commissioning of the ring. The kicker power supply was burnt due to heavy operation and old electronic components on July 5, 2011. So the commissioning was delayed a month to recover the kicker and check other components around the kicker power supply. We could begin the commissioning again and had succeeded a first accumulation on August 5, 2011. The storage current of $100 \mathrm{~mA}$ was achieved in the ring on October 7, 2011. The fist photons of the beam-lines also were checked on October 24, 2011. We did experiments with beam-line users to check the quality of the photons emitted from the ring on February 14, 2012. As a result, we started user service on March 21, 2012 as planned.

\section{Beamline}

The 3155 beam-line users (x-ray scattering, x-ray absorption spectroscopy, small angle xray scattering, crystallography, protein crystallography, x-ray micro-image, x-ray lithography, photoemission spectroscopy, magnetic spectroscopy, scanning photoemission microscopy, etc.) had visited our laboratory and conducted experiments more than 1000 in 2010 . During the first year of user service, we just had 78 users who did 18 experiments at two beam-lines. There were huge increases of users and proposals for 14 years. Figure 6 is showing a statistical chart of users and experiments conducted by them. There are 28 beam-lines in operation and 3 beamlines under construction just before upgrading PLS. One-third beam-lines were for surface and 
interface experiments using ultra violet range energy $(30-1100 \mathrm{eV})$. Two-third beam-lines were for x-ray experiments $(\sim 6-20 \mathrm{keV})$. Also there were four beam-lines built by outside institutes.

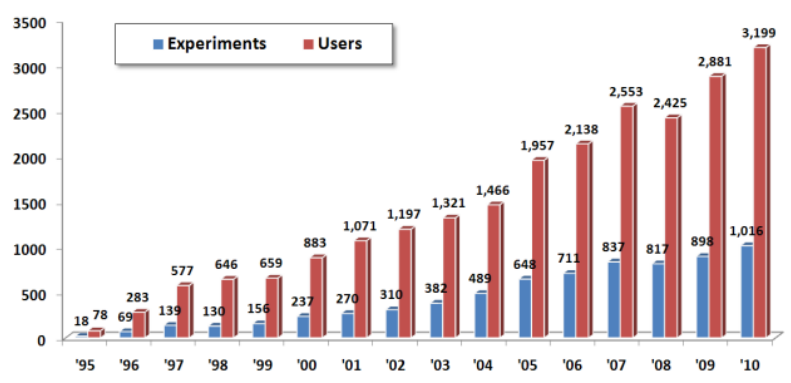

Figure 6. Statistic of beam-line users and proposals.

Figure 7 is the layout of PLSII beam-lines. There are 10 long straight sections and 10 short straight sections for insertion devices. And 20 bending magnets are used for beam-lines. We installed 30 beam-lines in 14 straight sections and 16 bending magnets, which are in operation (refer to website : http://pal.postech.ac.kr). Two insertion device beam-lines are under construction.

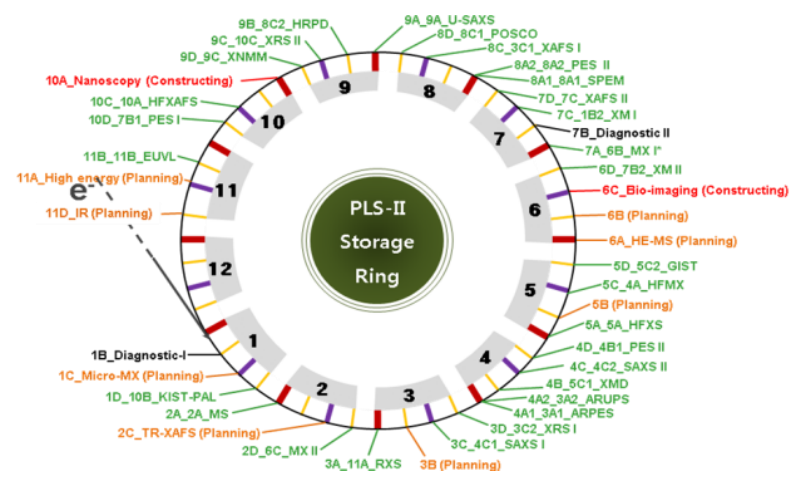

Figure 7. PLSII beam-line layout.

\section{Summary}

We upgraded PLSII Linac from 2.5 to $3.0 \mathrm{GeV}$ and commissioned it according to plan successfully. Now it is working as a $3.0 \mathrm{GeV}$ injector for the PLSII storage ring. And we achieved the first commissioning goal by getting $3.04 \mathrm{GeV}$ in energy (target value: $3.0 \mathrm{GeV}$ ), $150 \mathrm{~mA}$ in current (target value: $100 \mathrm{~mA}$ ), 10.5 hours in life time (target value: 10 hours) and $135 \mathrm{AH}$ (target value: 70 ampere hours) in beam dose during the commissioning.

In PLSII, we planned to change normal conducting RF cavities to super conducting cavities as shown in figure 8 . But there was an accident in delivering the first super conducting cavity. So a cavity was installed during the summer shutdown period. Now the operating beam current is $110 \mathrm{~mA}$ due to this reason. We will increase the beam current next year after installing the second super conducting cavity. Eventually, we had started first user service on March 21, 2012 and are consistently doing this service. 


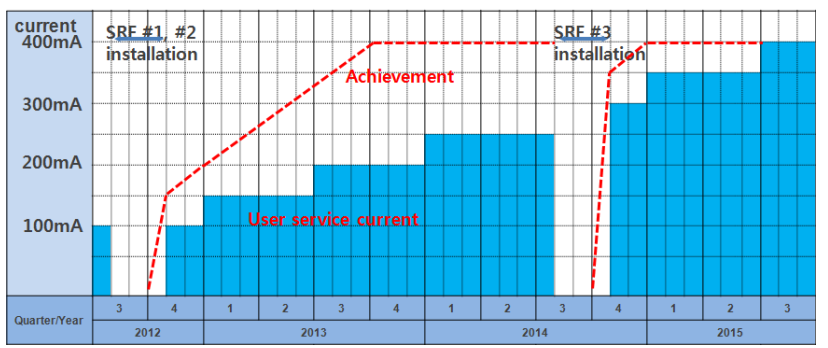

Figure 8. Milestone of changing RF cavities and supplying beam current for users.

\section{References}

[1] Pohang Accelerator Laboratory, "2010 PLS User Annual Report” (2011).

[2] K.R. Kim, "Current Status of PLS-II Upgrade Project: Accelerating System", the $14^{\text {th }}$ ICABU, October 7-8 (2010).

[3] R. B. Neal, ed., "The Stanford Two-Mile Accelerator," Q. A. Benjamin, New York (1968). 